\title{
Eficácia do tratamento com pressão negativa na cicatrização de lesão por pressão
}

\section{Effectiveness of treatment with negative pressure in the healing of lesions by pressure}

\author{
Eficacia del tratamiento con presión negativa en la cicatrización de lesión por presión
}

\author{
Michele Ribeiro', Verusk Arruda Mimura², Bruna Gabriela Bíbancos Damas², \\ Jessica Marques Ribeiro3 ${ }^{3}$,Tamiris Aparecida Silva ${ }^{4}$, Haialla Rezende Rodrigues ${ }^{5}$
}

\begin{abstract}
RESUMO
Trata-se de um relato de experiência sobre um paciente do sexo masculino internado em uma unidade de terapia intensiva apresentando uma lesão por pressão estágio 2 submetido à terapia por pressão negativa. O objetivo foi relatar a eficácia da terapia com pressão negativa no processo cicatricial de uma lesão. Os dados evolutivos foram obtidos por meio de avaliação dos registros fotográficos periódicos da lesão, respeitando-se os preceitos éticos e sendo, posteriormente, mensurados por meio do software Mobile Wound Analiser (MOWA). O tratamento baseou-se na aplicação de um curativo oclusivo acoplado a uma minibomba capaz de exercer uma pressão negativa contínua de $80 \mathrm{mmHg}$ no leito da lesão. O tempo da pesquisa foi de 14 dias com trocas da parte oclusiva do curativo num intervalo de 3 dias e da minibomba em 7 dias. Houve uma evolução nesse período culminando na cicatrização total no período de 14 dias. A terapia com pressão negativa favoreceu a circulação local e diminuiu o exsudato, proporcionando condições adequadas para acelerar a cicatrização.
\end{abstract}

DESCRITORES: Ferimentos e lesões. Tratamento de Ferimentos com Pressão Negativa. Úlcera por pressão. Estomaterapia.

\begin{abstract}
This is an experience report about a male patient admitted in an intensive care unit having a stage 2 lesion by pressure subjected to negative pressure therapy. The aim was to report the effectiveness of negative pressure therapy in the healing process of a lesion. Evolutionary data were obtained through evaluation of periodic photographic records of the lesion respecting the ethical precepts, and being subsequently measured by the Mobile Wound Analiser (MOWA) software. Treatment was based on the application of an occlusive dressing attached to a minipump capable of exerting a continuous negative pressure of $80 \mathrm{mmHg}$ in the wound bed. The length of the study was 14 days, with occlusive portion of the bandage exchanges with 3-day intervals and minipump exchange with an interval of 7 days. The patient progressed with complete healing in 14 days. The therapy using negative pressure favors the local circulation and decreased exudate, providing suitable conditions to accelerate healing
\end{abstract}

DESCRIPTORS: Wounds and injuries. Negative-pressure wound therapy. Pressure ulcer. Stomatherapy.

${ }^{1}$ Hospital São Camilo - Itapetininga (SP), Brasil.

Endereço para correspondência: Rua Dr. Coutinho, 628 - Centro - CEP: 18200-358 - Itapetininga (SP), Brasil - E-mail: miribeiro009@gmail.com

¿Universidade Paulista - Sorocaba (SP), Brasil.

${ }^{3}$ Hospital São Luiz - São Paulo (SP), Brasil.

${ }^{4}$ Hospital Samaritano - Sorocaba (SP), Brasil.

${ }^{5}$ Smith Nephew - Atibaia (SP), Brasil.

Artigo recebido em: 19/04/2017 - Aceito para publicação em: 21/08/2017 1 


\section{RESUMEN}

Se trata de un relato de experiencia acerca de un paciente del sexo masculino admitido en una unidad de cuidados intensivos presentando una lesión por presión nivel II, sometido a tratamiento por presión negativa. El objetivo fue describir la eficacia de la terapia con presión negativa en el proceso de curación de una lesión. Los datos evolutivos se obtuvieron mediante la evaluación de los registros fotográficos periódicos de la lesión, respetando los preceptos éticos, y analizados posteriormente por el software Mobile Wound Analiser (MOWA). El tratamiento se basó en la aplicación de un vendaje oclusivo acoplado a una minibomba capaz de ejercer una presión negativa constante de 80 mmHg en el lecho de la lesión. El tiempo del estudio fue de 14 días con cambios de la porción oclusiva del vendaje con intervalos de 3 días y de la minibomba con un intervalo de 7 días. El paciente ha progresado con la curación completa en 14 días. La terapia de presión negativa favorece la circulación local y la disminución de exudado, proporcionando condiciones adecuadas para acelerar la curación.

DESCRIPTORES: Heridas y lesiones. Terapia de Presión Negativa para Heridas. Úlcera por presión. Estomaterapia.

\section{INTRODUÇÃO}

Os pacientes internados em unidade de terapia intensiva (UTI) apresentam-se hemodinamicamente instáveis, com a mobilidade prejudicada em decorrência da própria doença ou como sequela dela. Por causa do seu estado geral comprometido, esses pacientes acabam, na maioria das vezes, adquirindo lesão por pressão, e todo esse quadro interfere no processo cicatricial da ferida ${ }^{1}$.

Devido às complexidades relacionadas ao processo cicatricial, cada vez mais novas tecnologias surgem para o tratamento de feridas. Uma dessas inovações é a terapia com pressão negativa, que obteve ampla repercussão nos últimos 15 anos. Seu advento sofreu várias modificações com o intuito de modernizar e tornar seu uso mais prático².

A pressão negativa é indicada em várias situações com objetivos distintos. Pode ser utilizada para preparar o leito da ferida para enxertos e fechamentos cirúrgicos ou ainda para promover a cicatrização por segunda intenção. Além de favorecer o processo cicatricial por acelerar a formação de tecido de granulação, reduz a colonização de bactérias, diminui a dor, o edema, o tempo de tratamento e, consequentemente, os custos em relação aos curativos convencionais existentes ${ }^{3}$.

O presente relato objetiva caracterizar a evolução do processo cicatricial de uma lesão por pressão estágio $2 \mathrm{em}$ um paciente internado na UTI submetido à terapia negativa.

\section{MÉTODOS}

Trata-se de estudo descritivo, tipo relato de caso, realizado na UTI de um hospital geral no interior de São Paulo no período de 10 a 24 de setembro de 2015. O projeto foi aprovado pelo Comitê de Ética em Pesquisa da Associação Unificada Paulista de Ensino Renovado Objetivo, processo CAAE no 45340115. 0.0000.5512, parecer no 1.103.783, conforme preconiza a Resolução no 466/2012 do Conselho Nacional de Saúde. Obteve-se, ainda, o consentimento livre e esclarecido do paciente. Os dados foram coletados a partir de observação, avaliação e acompanhamento da evolução da lesão. Adotou-se o critério de seleção do paciente para pesquisa de acordo com a classificação da lesão, sendo requisito para escolha possuir lesão por pressão estágio 2 .

O paciente selecionado foi submetido ao tratamento com pressão negativa por um período de 14 dias, no qual foram necessários dois kits de curativos com quatro trocas de coberturas e duas minibombas de pressão negativa, sendo que cada substituição de coberturas foi realizada a cada três ou quatro dias e a minibomba a cada sete dias. .

$\mathrm{Na}$ instituição em que foi a realizada a pesquisa, o enfermeiro tem por função fazer as avaliações e prescrever o curativo para o tratamento de lesões. Assim sendo, tal avaliação ocorreu anteriormente e durante a aplicação do dispositivo portátil de pressão negativa de uso único, capaz de exercer pressão negativa de $80 \mathrm{mmHg}$, descartável, com uso contínuo por 14 dias. A reavaliação do paciente aconteceu em cinco momentos distintos:

- aplicação do dispositivo de pressão negativa (1º dia);

- troca do curativo oclusivo ( $4^{\circ}$ e $11^{\circ}$ dias);

- troca da minibomba do sistema de pressão e curativo oclusivo ( $\left.7^{\circ} \mathrm{dia}\right)$; e

- retirada do curativo de pressão negativa (14\% dia).

Para mensurar a área de dimensão (largura x altura) da úlcera $\left(\mathrm{cm}^{2}\right)$, esta foi fotografada antes da aplicação e no 
momento de cada troca de curativo, com a utilização do software Mobile Wound Analiser (MOWA ).

\section{RELATO DO CASO}

Paciente de 49 anos, do gênero masculino, acamado, procurou a unidade de pronto atendimento local no dia 5 setembro de 2015, às 18 horas, referindo diarreia e vômito, segundo informações colhidas, com evolução para uma crise convulsiva com hipotensão, que resultou em uma parada cardiorrespiratória. Durante a madrugada foi encaminhado para a UTI e permaneceu com intubação orotraqueal, sonda vesical de demora, com diagnóstico clínico de encefalopatia hepática, infecção no trato urinário e pancreatite. Ao exame físico, apresentou os seguintes sintomas: levemente descorado, desidratado, ictérico e febril, normocárdico, normotenso, ausculta cardíaca com presença de bulhas rítmicas normofonéticas 2 tempos sem sopros audíveis, ausculta pulmonar com murmúrios vesiculares presentes sem ruídos adventícios, abdome distendido com ruídos hidroaéreos presentes, lesão por pressão (LP) estágio 2 em região sacral, membros inferiores edemaciados.

Após a estabilização do paciente, iniciou-se o procedimento de tratamento da lesão por pressão com a aplicação de curativo de pressão negativa na UTI. A equipe de enfermagem recebeu orientação quanto aos cuidados com o dispositivo portátil de pressão negativa (não desligar e não molhar a minibomba), e quanto ao intervalo de permanência dos curativos entre as trocas e a mudança de decúbito, que deveria ocorrer com a mesma frequência.

\section{RESULTADOS}

No momento da aplicação do curativo, D+0 (dia do início do tratamento com pressão negativa), o paciente possuía uma LP na região sacral estágio 2, que apresentava uma extensão de 4,8 x 7,1 cm e área de $11,3 \mathrm{~cm}^{2}$. A lesão mostrou-se colonizada, pouco exsudativa (característica sanguinolenta) A pele perilesional estava rosa e descamativa. Não havia presença de edema ou odor.

$\mathrm{Na}$ primeira troca de curativo, no $\mathrm{D}+4$ (4 dias após o início do tratamento com o dispositivo de pressão negativo ultraportátil), a lesão apresentou a extensão de 4,0 x 7,1 cm com área de $9,8 \mathrm{~cm}^{2}$, com evidência de aumento do tecido de epitelização de aproximadamente 13,27\%, reduzindo a dimensão da ferida. Observou-se ainda uma diminuição significativa do exsudato sanguinolento observado anteriormente.

No D+7, a extensão da lesão era 2,0 x 3,2 cm e a área, de $2,8 \mathrm{~cm}^{2}$, apresentando um aumento do tecido epitelizado de 75,22\% em relação à dimensão da lesão antes da aplicação do dispositivo de pressão negativo ultraportátil.

No D+10 o paciente foi encaminhado à enfermaria e passou a ser acompanhado constantemente por um familiar. Devido à falha na comunicação sobre o manejo e cuidados com o curativo entre equipe de enfermagem e acompanhante, houve uma interferência no tratamento com a pressão negativa, pois este inadvertidamente desligou o dispositivo de pressão negativa, resultando na interrupção do tratamento da ferida no leito por um período indeterminado. No D+11, constataram-se apenas duas pequenas áreas de lesão, com dimensões 0,2 x 0,6 cm (área de 0,2 $\mathrm{cm}^{2}$ ) e 0,3 x 0,6 cm (área de 0,2 $\mathrm{cm}^{2}$ ). Comparando-se as áreas dessas lesões com aquela apresentada antes do início do tratamento, pôde-se notar uma melhora de 95,4\%. No D+14, o tratamento foi finalizado devido à cicatrização de $100 \%$ da LP (Figuras 1 e 2).

\section{DISCUSSÃO}

Familiares relatam que o paciente adquiriu essa lesão em uma internação anterior e já estava aproximadamente há um mês e meio realizando curativos com ácidos graxos essenciais (AGE) em sua residência.

Em pesquisa realizada anteriormente por outros autores, constatou-se que uma LP estágio 2 apresenta cicatrização total em 35 semanas quando aplicada uma gaze umedecida em soro fisiológico (SF) 0,9\% nas trocas diárias, assim como a espuma de poliuretano e apresenta o mesmo período de cicatrização do SF 0,9\%, já o hidrocoloide aplicado após a limpeza da lesão, com troca duas vezes por semana, é capaz de promover a cicatrização em 8 semanas em 50\% dos casos 4 .

Observou-se que o mesmo paciente apresentou mobilidade prejudicada devido à lesão estar localizada em região de proeminência óssea, que torna a cicatrização complexa e muitas vezes lenta. Foi constatada em cada troca a diminuição gradativa da ferida (Figura 2) e, mesmo com a intercorrência no décimo dia de tratamento com o curativo de pressão negativa, foi possível observar uma diminuição de $2,4 \mathrm{~cm}^{2}$ da lesão em relação à troca anterior. 
Isso nos permite afirmar que a terapia com o uso de pressão negativa favoreceu a circulação local e diminuiu o exsudato, proporcionando condições adequadas para acelerar a cicatrização.
Outros autores já afirmaram que a pressão negativa vem sendo muito utilizada como adjuvante nos últimos anos para o tratamento de feridas complexas como úlceras por pressão. Os mecanismos de ação da pressão negativa
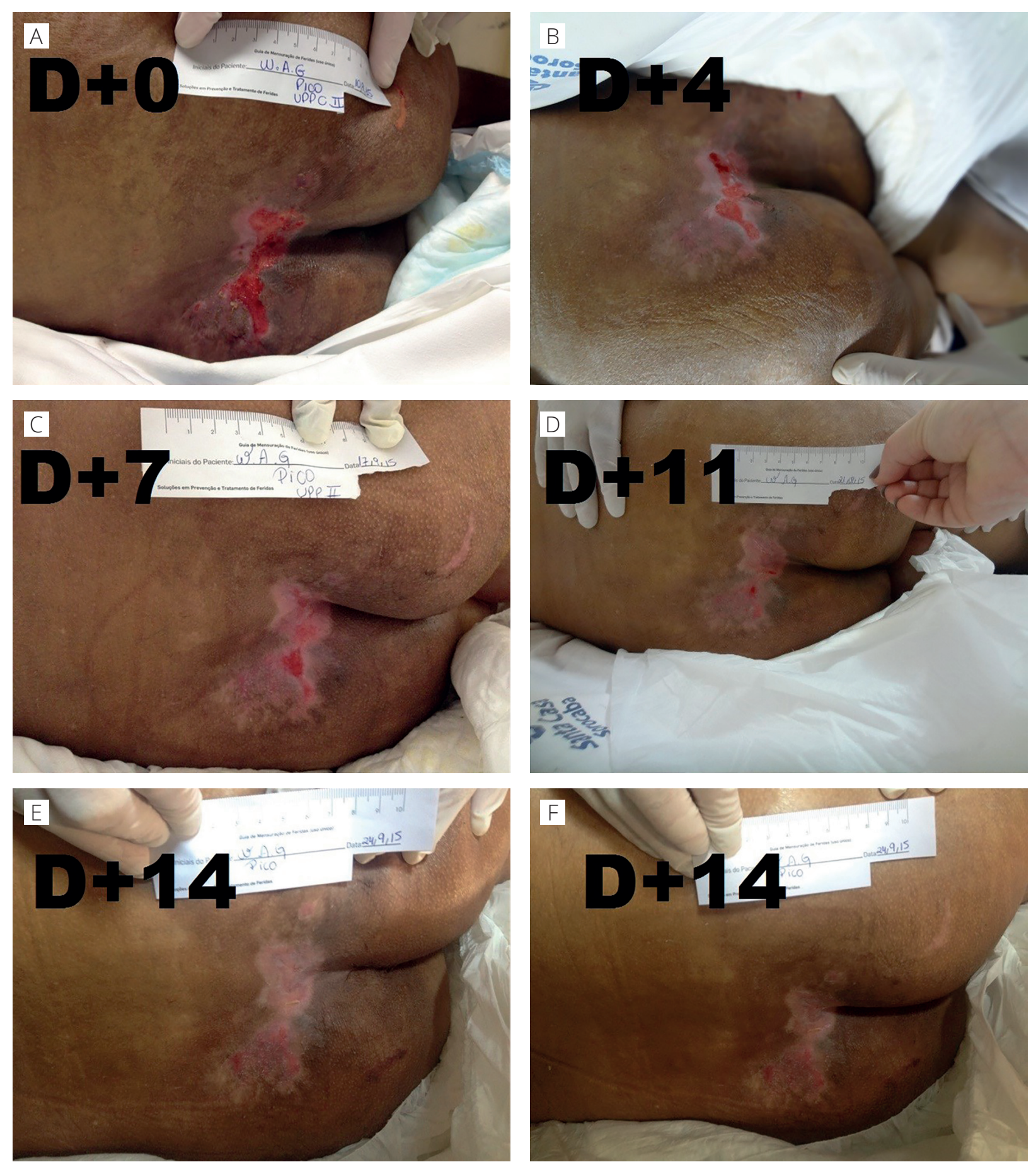

Figura 1. Fotografias da evolução do tratamento da lesão por pressão estágio 2 , em região sacral com a terapia de pressão negativa no dia do início do tratamento $(D+0)$; quarto dia após o início do tratamento e dia da primeira troca de curativo $(D+4)$; sétimo dia após o início do tratamento e dia da segunda troca de curativo ( $D+7)$; décimo primeiro dia após o início do tratamento e dia da terceira troca de curativo $(\mathrm{D}+11)$; e décimo quarto dia após o início do tratamento e dia da retirada do curativo (D+14). 
nas feridas se baseiam na interação de cinco mecanismos diferentes:

1. aumento no fluxo sanguíneo local;

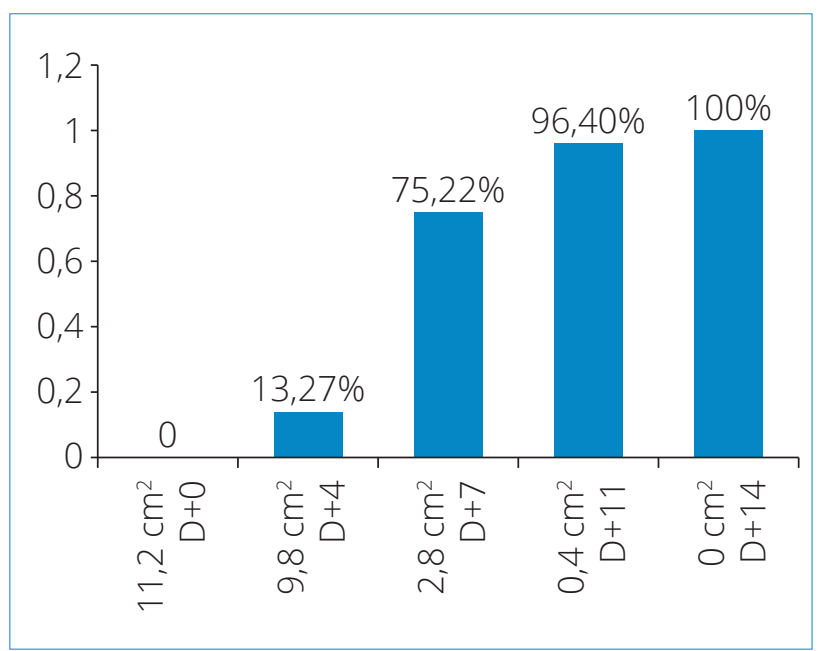

Figura 2. Representação gráfica da evolução da cicatrização de úlcera por pressão tratada com terapia de pressão negativa, em percentual de área cicatrizada, por dias de tratamento. As áreas da úlcera foram mensuradas e calculadas a partir da análise das imagens pelo software Mobile Wound Analiser (MOWA).
2. remoção de fluidos e redução do edema;

3. microdeformação do leito da ferida resultando em estímulo da proliferação celular;

4. macrodeformação atuando como um expansor tecidual;

5. manutenção da homeostase local, com estímulo da angiogênese e tecido de granulação ${ }^{5}$.

\section{CONCLUSÃO}

Para as autoras, o presente trabalho possibilitou a ampliação do conhecimento e a experiência relacionada à utilização da terapia com pressão negativa no processo cicatricial. A literatura nacional revelou-se escassa no que se refere ao emprego desse tipo de tratamento, evidenciando a necessidade de realização de mais pesquisas voltadas para o assunto em questão. Com base nos resultados apresentados, conclui-se que o tratamento da úlcera por pressão realizado com a terapia de pressão negativa é muito eficiente, evoluindo com uma evidente aceleração do processo cicatricial do paciente observado.

\section{REFERÊNCIAS}

1. Fernandes NCS, Torres GV, Vieira D. Fatores de risco e condições predisponentes para úlcera de pressão em pacientes de terapia intensiva. Rev Eletr Enf [Internet]. 2008 [citado em ago. 2016];10(3):733-46. Disponível em: https:// www.fen.ufg.br/fen_revista/v10/n3/pdf/v10n3a19.pdf

2. Birke-Sorensen H, Malmsjo M, Rome P, Hudson D, Krug E, Berg $L$, et al. Evidence-based recommendations for negative pressure wound therapy: treatment variables (pressure levels, wound filler and contact layer) - steps towards an international consensus. J Plast Reconstr Aesthet Surg. 2011 [citado em ago. 2016];64(Suppl):S1-16. Disponível em: https://www.ncbi.nlm.nih.gov/pubmed/21868296
3. Hudson, da., Adams, Koehler, Van Huyssteen, A., Martin, R. E Huddleston, E.M. (2015). Terapia de ferida de pressão negativa simplificada: avaliação clínica de um uktraportable, sistema de não-vasilha. Ferida int J. Ferida int J. 2011; 12:195. Disponível em: https://www. NCBI.nlm.nih.gov/m/PubMed/23647737 doi: 10.1111/ iwj. 12080

4. Rosa TJS, Cintra LKL, Freitas KB, Alcântara PFDL, Spacassassi F, Rosa CDP, et al. Úlceras por pressão: tratamento. Acta Fisiatr. 2013;20(2):106-111.

5. Wada A, Teixeira Neto N, Ferreira MC. Úlceras por pressão. Rev Med (São Paulo). 2010;89(3/4):170-7. 